Journal of Computer Science 7 (12): 1831-1838, 2011

ISSN 1549-3636

(C) 2011 Science Publications

\title{
Improving Diagnostic Viewing of Medical Images using Enhancement Algorithms
}

\author{
Hanan Saleh S. Ahmed and Md Jan Nordin \\ School of Computer Science, \\ Faculty of Information Science and Technology, \\ Universiti Kebangsaan Malaysia, Selangor, Malaysia
}

\begin{abstract}
Problem statement: Various images are low quality and difficultly to detect and extract information. Therefore, the image has to get under a process called image enhancement which contains an aggregation of techniques that look for improving the visual aspect of an image. Medical images are one of the fundamental images, because they are used in more sensitive field which is a medical field. The raw data obtained straight from devices of medical acquisition may afford a comparatively poor image quality representation and may destroy by several types of noises. Image Enhancement (IE) and denoising algorithms for executing the requirements of digital medical image enhancement is introduced. The main goal of this study is to improve features and gain better characteristics of medical images for a right diagnosis. Approach: The proposed techniques start by the median filter for removing noise on images followed by unsharp mask filter which is believable the usual type of sharpening. Medical images were usually poor quality especially in contrast. For solving this problem, we proposed Contrast Limited Adaptive Histogram Equalization (CLAHE) which is one of the techniques in a computer image processing domain. It was used to amend contrast in images. Results: For testing purposes, different sizes and various types of medical images were used and more than 60 images in different parts of the body. From the experts' evaluation, they noted that the enhanced images improved up to $80 \%$ from the original images depends on medical images modalities. Conclusion: The proposed algorithms results were significant for increasing the visibleness of relatively details without distorting the images.
\end{abstract}

Key words: Medical images, median filter, unsharp mask, contrast limited adaptive histogram equalization, Medpix database

\section{INTRODUCTION}

Image Enhancement (IE) is essentially improving the perception or interpretation of image information for supplying better input data for other image processing techniques. IE transforms images to provide better representation of the subtle details. It is an indispensable tool for researchers in a wide variety of fields including (but not limited to) medical imaging, art studies, forensics and atmospheric sciences. It is application specific: An IE technique suitable for one problem might be inadequate for another. For example forensic images or videos employ techniques that resolve the problem of low resolution and motion blur while medical imaging benefits more from increased contrast and sharpness. Is the process of manipulating images so that the output image is more suited than original for a spatial application? Images are taken by different devices, so, a method which is quite effective for enhancing satellite images taken in the infrared band of the electromagnetic spectrum (Gonzalez and Woods,
2008) may not be the best way for enhancing x-ray images. There is no common or specific "theory" for enhancing images. When images are processed for visual interpretation, viewers are the ultimate judge of how well a specific method works.

Thereare several techniques for enhancing digital images without spoiling it. The enhancement techniques can generally be classified in to the following two classes:

- Spatial domain methods

- Frequency domain methods

In spatial domain methods, we directly deal with the pixels of image. The pixel values are manipulated to achieve coveted enhancement. In frequency domain techniques, the image is first transferred in to frequency domain. It means that, the Fourier Transform of the image is computed first. All operations ofimage enhancement are executed on the Fourier transform of

Corresponding Author: Hanan Saleh, School of Computer Science, Faculty of Information Science and Technology,

Universiti Kebangsaan Malaysia, Selangor, Malaysia 
the image and then the Inverse Fourier transform is executed to obtain the resultant images. These enhancement operations are performed in order to modify the image brightness, contrast or the distribution of the grey levels. As a consequence the pixel value (intensities) of the output image will be modified according to the transformation function used in several domains where images should be analyzed and understood. For example, satellitesimages analysis, analysis of images from medical devices etc.

Medical images modalities: The dawn of the modern era of medical diagnosis can be traced to 1896, when the first $\mathrm{x}$-ray has been captured by Wilhelm Roentgen, which was of his wife's hand. The development of radiology grew rapidly after that. Later, modern x-ray techniques have been developed to significantly improve both the contrast detail and the spatial resolution. Better image details let the smaller places diagnosis of pathology than may be discovered by older technologies. Radioscopy is a flow of medical science that treats with medical modalities. X-ray devices or other such radiation machines are utilized for imaging purpose. Radiology also includes techniques that do not require radiation, such as ultrasound and MRI. Radiology can be divided into two streams which are diagnostic radiology and therapeutic radiology. Diagnostic radiology is interested with using different imaging modalities to serve in disease diagnosis. Diagnostic radiology can be further divided into multiple sub-specialty domains. Interventional radiology is one of the sub-specialty areas which use the imaging modalities of diagnostic radiology to guide minimally invasive surgical procedures. On the other hand, Therapeutic radiology, also named radiation oncology uses radiation to treat diseases like cancer. Some of the popular imaging modalities are:

- General radiography

- Magnetic Resonance Imaging (MRI)

- Ultrasound

- Computed Tomography (CT)

- Nuclear imaging techniques

General radiography: In this imaging modality, $x-$ rays are used to create images and for the evaluation of several soft tissue and bony structures. Angiography and fluoroscopy are some of the spatial applications of $\mathrm{X}$-ray imaging which have real-time imaging ability.

CT scanning: This imaging technique utilizes X-rays in conjunction with computing algorithms to image tissues in the body. At times, contrast media is utilized to delineate anatomy and allow 3D reconstructions of structures, such as veins and arteries.

Sonography: Medical ultrasonography uses highfrequency sound waves to visualize soft tissue structures in the body in real time and does not involve any radiation.

Magnetic Resonance Imaging (MRI): It is a noninvasive medical test that utilizes a powerful magnetic field, radio frequency pulses and a computer to produce detailed images of organs, soft tissues, bone and virtually all other internal body structures for treatment and diagnosis.

Nuclear medicine: This imaging technique involves the use of radionuclides or radiopharmaceuticals for evaluation of lungs, heart, liver, thyroid, bones and gallbladder for diagnosis and treatment. Radiologists utilize these imaging technologies for diagnosis and treatment of various diseases.

Related work: Tsai et al., 2002; Chen et al., 1994; Dawood et al., 2011; Achmad et al., 2011; Jaya and Thanushkodi, 2011; Mohammed et al., 2009; Ramos and Tahan, 2009; Ping et al., 2006 used IE methods for sharpening details and reducing noise for improving the visual representation of medical images. Since minute details play a decisive part in diagnosis and treatment of disease, it is necessity to highlight significant features while displaying medical images. This makes IE an essential tool to view anatomic region in ultrasound, $\mathrm{x}$ rays and MRI.

Jagatheeswari1 et al., 2009 used a median filter $(3 \times 3$ mask $)$ with histogram equalization. After Minimum Mean Brightness Error Bi-Histogram Equalization (MMBEBHE) method applied on the images, the impulse noise appeared in the images. To avoid this effect, the enhanced image has been passed through a median filter. Also used a median filter to smooth the images before border detection, but with a big enough neighborhood to smooth out artifacts such as hair and air bubble.

Frosio and Borghese, 2006 presented that the unsharp mask technique is one of the common utilized methods for enhancing x-ray images. They defined that UnSharp Mask filter (USM) can be easy applied for filtering in real time. Furthermore, incephalometric radiography it enhances the small features visibilities, underexposed bony characteristics. Sen et al. 2002 applied unsharp filter had exhibited that it can guide to artifacts such as edge overshoot that can make clipping of secondary vascular structures near larger.

The new histogram is different from the ordinary histogram, because the intensity of each pixel is limited 
to a user-selectable maximum. So CLAHE can limit the noise in images. To overcome the drawback and noise amplification, CLAHE which is a generation of Adaptive Histogram Equalization (AHE) method was introduced by Pizer et al., 1990. In addition, Parveen and Sathik 2009 have enhanced bone fracture images by contrast limited adaptive histogramequalization. The results have shown that CLAHEgives better result than other Equalization.

\section{MATERIALSANDMETHODS}

Overview: There are a lot of techniques used in image enhancement or restoration including low pass filtering, high pass filtering, sobel edge filtering, median filtering, histogram equalization and its various. Proposed method consists of three steps as following:

- Median filter for noise reduction

- UnSharp Mask filter (USM) for edges sharpening

- Contrast Limited Adaptive Histogram Equalization (CLAHE) for contrast enhancement

Median filter for noise reduction: Filtering is a part of image enhancement which is used to enhance certain details such as edges in the image that are relevant to the application. In addition to that, filtering can also be used to eliminate unwanted elements of noise. Medical images usually contain salt and pepper noise. This noise appears due to the presence of minute gray scale variations in the image. Median filtering is a popular technique of the image enhancement for removing impulse noisewithout effectively reducing the image sharpness (Chan et al., 2005).

Median filter is quite common because it provides excellent noise-reduction abilities, with basically less blurring than similar size linear smoothing filters. Here, the median process was performed by sliding a $3 \times 3$ windowing operator over the image. It considers every pixel and its neighbors in images to find out whether or not it is a representation of the surroundings. It replaces the value of pixel with the median of the neighboring pixel elements. We calculated the median by sorting the whole pixel values from the neighborhood into numeral sort and then replaced the pixel being studied with the middle pixel value. If the locality under conditionconstitutes an even pixels value, the average of the two middle pixel values is the median.

Unsharpmask filter for edges sharpening: Unsharpfiltering is an uncomplicated sharpening process that gains its name from the study which it improves edges and other high frequency components in images through a process that deducts a smoothed or unsharp version of images from the input images. In our study, the use of the classical unsharp masking filter after median filter to reduce of the remained noise and sharpen the edges. Firstly it is obtained a blurred form of the original image. This is carried out by applying the low-pass filter, in our case Gaussian blur algorithm using a small radius. We used a two pixel radius and applied Gaussian blur filter only two times. The blurred form of the image is then pixel deducted from the original image and so it is obtained the high pass component. The output image is obtained by adding the high-pass component to the original image. Because the output image could contain also pixels with negative values, it is then normalized. No threshold cutoff was used. The two steps for the unsharp mask filter are mentioned below:

- Unsharp mask filter creates edge images $\mathrm{g}(\mathrm{x}, \mathrm{y})$ from input images $\mathrm{f}(\mathrm{x}, \mathrm{y})$ in this Eq. 1 .

$$
g(x, y)=f(x, y)-f_{\text {smooth }}(x, y)
$$

Where, $f_{\text {smooth }}(x, y)$ is a smoothed form of $f(x, y)$ (Gaussian blur algorithm) as shown in Fig. 1.

- The edge images from the result of subtracting input images from low pass signal could be utilized for images sharpening by adding it backward into the input signal, as illustrated in Fig. 2.

This function is represented as follows:

$f_{\text {sharp }}(x, y)=f(x, y)+k^{*} g(x, y)$

Where, $\mathrm{k}$ is a scaling constant, values for $\mathrm{k}(\mathrm{k} \geq 0)$, for generally. When $\mathrm{k}>1$, the process is referred to as highboost filtering. In our process, we have applied $\mathrm{k}=1$.

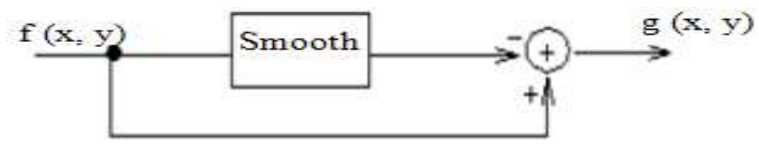

Fig. 1: Spatial sharpening

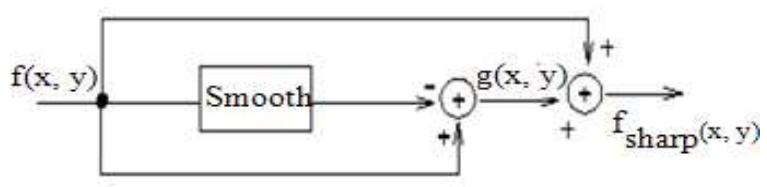

Fig. 2: The complete unsharp filtering operator 
The basic advantage of the unsharp filtering over other sharpening filters is the control flexibleness, because a vast majority of other sharpening filters do not supply any user-adjustable parameters. Unsharp filtering as other filters enhances fine detail and edges in digital images.

\section{Contrast Limited Adaptive Histogram Equalization}

(CLAHE): Contrast limited adaptive histogram is a technique utilized for improving the local contrast of images. It is a generalization of ordinary histogram equalization and adaptive histogram equalization.

CLAHE does not operate on the whole image works like ordinary Histogram Equalization (HE), but it works on small areas in images, named tiles. Each tile's contrast is enhanced, so that the histogram of the output area roughly matches the histogram determined by the 'Distribution' parameter. The adjacent tiles are then combined using bilinear interpolation to eliminate artificially induced boundaries. The contrast, particularly in homogeneous regions, can be limited to avoid amplifying any unwanted information like noise which could be exist in images.

Problems associated with HE and AHE can be limited by reducing contrast enhancement particularly in homogeneous areas. These regions can be featured by a high peak in the histogram connected with the contextual areas since several pixels fall inside the same gray level scope. The algorithm (Contrast Limited Adaptive Histogram Equalization (CLAHE)) limits the slope associated with the gray level assignment scheme to prevent saturation. This process is accomplished by allowing only a maximum number of pixels in each of the bins associated with the local histograms. After "clipping" the histogram, the clipped pixels are equally redistributed over the whole histogram to keep the total histogram count identical.

The CLAHE method can be divided into steps (Xu et al., 2009) to achieve as following:

- The Medical image is divided into contextual regions which are continuous and non-overlapping. Each contextual region size is $\mathrm{M} \times \mathrm{N}$ (the contextual regions size was set here to $8 \times 8$ )

- The histograms of each contextual regions are calculated

- The histograms of each contextual regions are clipped (A clip limit was set here to 0.01)

The pixels number in the contextual region is equally, distributed to each gray level. Then the average number of pixels in each gray level is defined as follows:
$\mathrm{N}_{Q V}=\frac{\mathrm{N}_{C R-\mathrm{R}} \times \mathrm{N}_{C R-\gamma}(3)}{\mathrm{N}_{\mathrm{g}}}$

Where:

$\mathrm{N}_{\mathrm{av}}=$ Average number of pixels

$\mathrm{N}_{\mathrm{g}}=$ Gray levels number in the contextual region

$\mathrm{N}_{\mathrm{CR}-\mathrm{x}}=$ Pixels number in the $\mathrm{x}$ dimension of the contextual region

$\mathrm{N}_{\mathrm{CR}-\mathrm{y}}=$ pixels number in the $\mathrm{y}$ dimension of the contextual region

Base on the Eq. 3, the $\mathrm{N}_{\mathrm{ac}}$ can be calculated by the eq. 4:

$\mathrm{N}_{\mathrm{ac}}=\mathrm{N}_{\mathrm{c}} \times \mathrm{N}_{\mathrm{av}}$

Where, $\mathrm{N}_{\mathrm{ac}}$ is actual clip-limit; $\mathrm{N}_{\mathrm{c}}$ is the maximum multiple of average pixels in each gray level of the contextual region.

The original and clipped histograms are shown in Fig. 3. In Fig. 3(a) if the number of pixels is greater thanN $N_{c}$, the pixels will be clipped. The total number of clipped pixels is defined as $\mathrm{N}_{\Sigma c}$, and then the number of pixels distributed averagely into each gray level is given by Eq. 5:

$N_{\mathrm{arp}}=\frac{N_{\mathrm{Z} z}}{N_{g}}$

After the above distribution, the remaining number of clipped pixels is expressed as $\mathrm{N}_{\mathrm{LP}}$ and then the step of distributed pixels is given by

$P_{d}=\frac{N_{g}}{N_{L P}}$

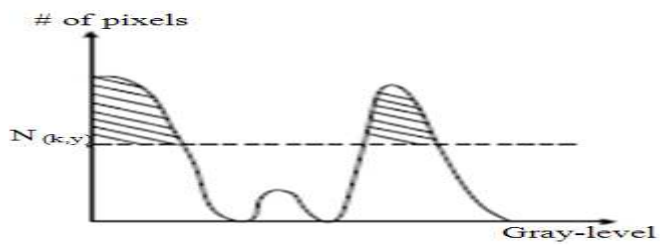

(a)

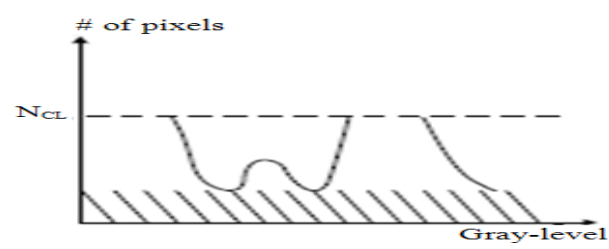

(b)

Fig. 3: Original and clipped histograms (a): original histogram (b): clipped histogram 
The algorithm work starts search according to the above step from the minimum gray level to the maximum. If the pixels number in the gray level is less than $\mathrm{N}_{\mathrm{ac}}$, the algorithm will distribute one pixel to the gray level. If the pixels are not all distributed when the search is end, the algorithm will calculate the new step according to Eq.6 and start new search until the remaining pixels is all distributed. Then, the new histogram in Fig. 3b is achieved.

- The grayscale histogram which is limited contrast in each contextual region is processed by histogram equalization. In our study, the Bins number for the histogram building for contrast enhancing transformation is restricted to 256 and the distribution of histogram is 'uniform' or flat histogram. In this study, the range is not specified in the experiment to get the full range of output image

- The points in the middle of the contextual region are considered as the sample points

- The result mapping at any pixel is interpolated from the sample mappings at the four surrounding samplegrid pixels.Pixels in the borders of the image outside of the sample pixels need to be processed specially. The neighboring tiles were combined using bilinear interpolation and the gray scale values were altered according to the modified histograms

The main advantages of the CLAHE transform are its modest computational requirements, ease of use and excellent results on most images.

\section{RESULTS}

The proposed methods have applied on different parts of the body. Some results are illustrated below:

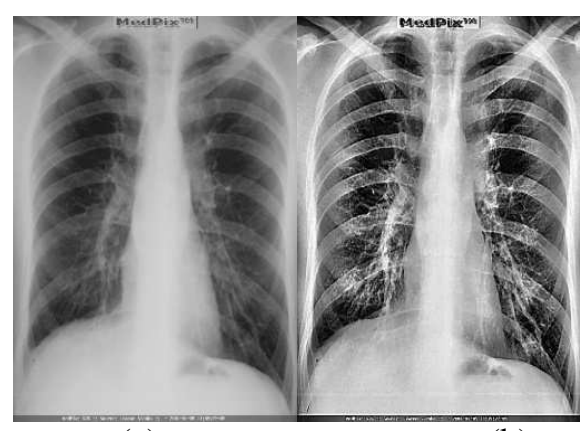

(a)

(b)

Fig. 4: X-ray image (a) original image, (b) after proposed methods

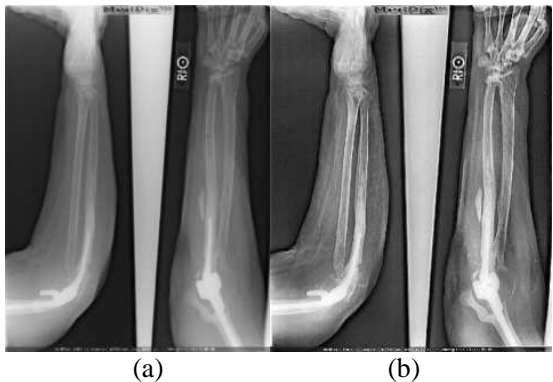

Fig. 5: X-ray image (a) original image, (b) after proposed methods

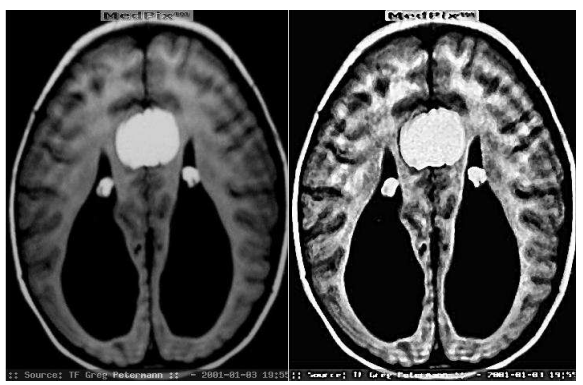

(a)

(b)

Fig. 6: MRI image (a) Original image, (b) after proposed methods

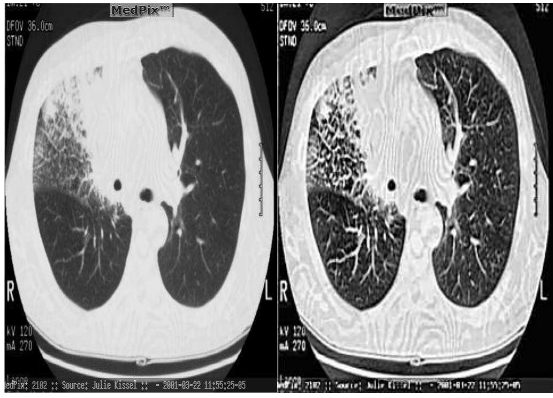

(a)

(b)

Fig. 7: CT image (a) Original image, (b) after proposed methods

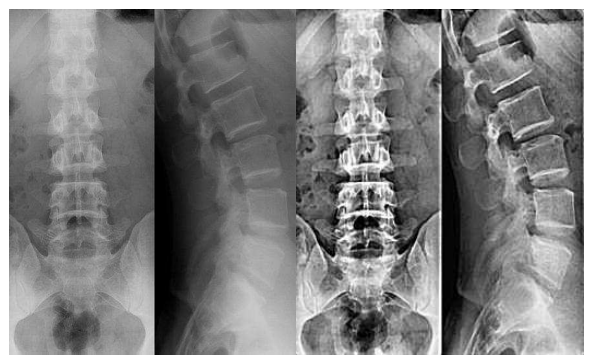

(a)

(b)

Fig. 8: X-ray image (a) original image, (b) after proposed methods 


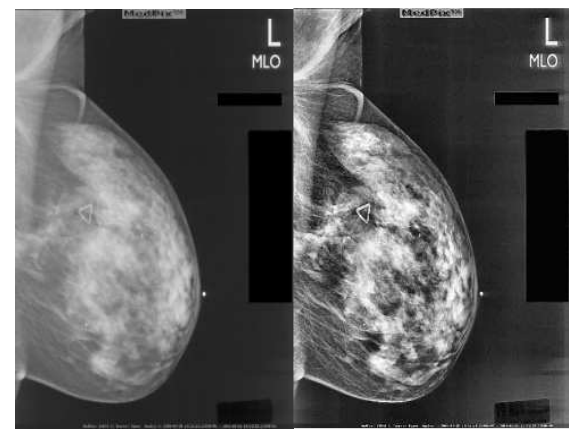

(a)

(b)

Fig. 9: Mammogram image (a) original image, (b) after proposed methods

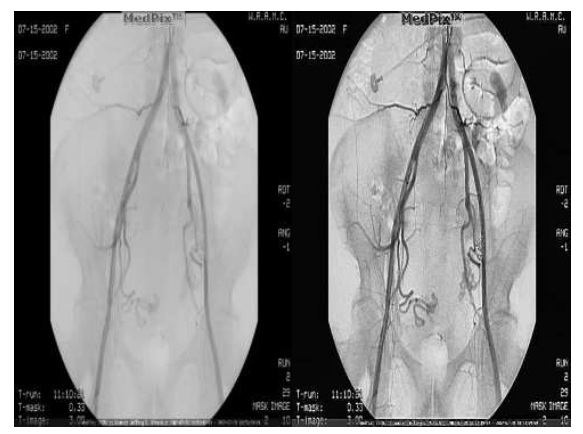

(a)

(b)

Fig. 10: Angiogram image (a) original image, (b) after proposed methods

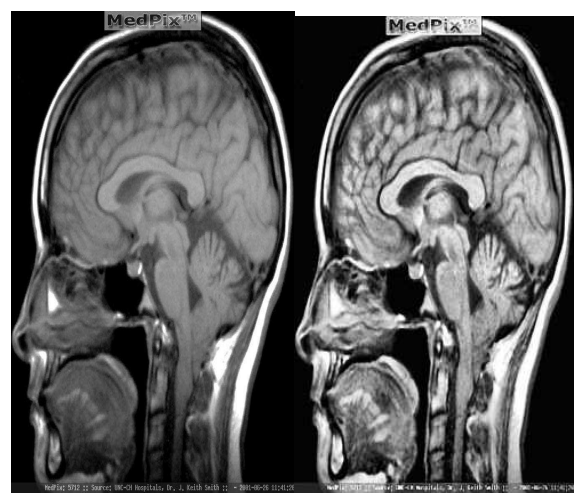

(a)

(b)

Fig. 11: MRI images (a) original image, (b) after proposed methods

\section{DISCUSSION}

Enhancement techniques play an significant role in enhancing medical images quality. Several previous results have been proving that contrast enhancement techniques capable to enhance the images' brightness and clean up unwanted noises. We have presented here effective algorithms for enhancement of images received from imaging devices. The medical images used as the inputs were collected from the medical images database website (MedPix Database) Medical Image Database, (2011).

The first process was noise reduction; median filter has been applied on various medical images for removing high frequency components such as impulsive noise, salt and pepper noise, etc as shown in Results Section. The results have proved that median filter can remove impulsive noise from several medical images without disturbing of the edges. In this enhancement stage, the median filtering was applied for each pixel of a $3 \times 3$ window of neighborhood pixels were extracted and analyzed the mean gray value of foreground, mean value of background and contrast value.

The second process was edge sharpening, we can see that unsharp mask filter was very effective on medical images for example it has shown increasing edge enhancement of fine detail such as the trabecular pattern of the bones as shown in Fig. 4, 5 and 8. Also it has improved and sharpened edges of tissue such as MRI image in Fig. 6 and Fig. 11, CT in Fig. 7. Fig. 1 and 8 prove that how much unsharp mask filter was significant on chest bones and on spines respectively.

One of the problems which medical images are suffering from is low contrast. Contrast enhancement should have the single goal of transmitting information in the image data most effectively; that is making intensity differences increase with the importance of the differences. Here, we have combined the results of medical filter and unsharp mask as input data to the contrast limited adaptive histogram equalization algorithm. Contrast at any position in an image is perceived in relation to local image context and not to the whole image. Hence, at each position in an image, displayed intensity should adapt to the local intensity distribution. That is, the foremost property of a contrast enhancement method should be that for each point in the image, the resulting intensity should depend on a region centered at that point. We call this the contextual region of the point. CLAHE has been shown to be very effective for a wide range of medical images (results above compared with the original images). Included are CT images, where the effect is especially striking in studies in which it is important to appreciate contrast simultaneously in different tissue types; MRI images, in which the effect is especially useful for surface coil images because of the correction for non homogeneity of sensitivity with depth; portal film images from radiotherapy in which the low contrast can be strikingly improved; and a wide range of radiographs.In addition, vessels have become more visibleon Mammogram and Angiogram images (see Fig. 9 and Fig. 10). 
Results evaluation: Our result was evaluated by experts. The experts' evaluation has been chosen because doctors are the best category cancheck if the proposed algorithms give better result for right diagnosis compared with the original data. We have sent more than 60 images in different parts ofbody and various medical imaging to experts. From the experts' evaluation, it is clearly that the proposed algorithms improve the appearance of image details significantly nearly in the most input images. They have demonstrated that the enhancement which our proposed algorithms have made are quite impressive for example bone and soft tissue density are more clearer and they can extract the information easily especially in images which are more darker or whiter. The expert mentioned that the improvement that happened in all parts of the body and in different medical imaging, but some improvements are much significant than others as mentioned in Discussion Section.The expert has presented that the enhancement of images can reachedup to $80 \%$ from the original images and this result depends on medical images modalities.

\section{CONCLUSION}

In the proposed method, we have enhanced medical images by effective enhancement algorithms which are median filter, unsharp mask filter and contrast limited adaptive histogram equalization. The proposed methods have been implemented by Matlab and more than 60 medical images in different parts of the body have been used to evaluate the performance of the proposed methods. The improvement of input medical images is up to $80 \%$ depends on medical images modalities and this result based on experts' evaluation by comparing input and output images.

\section{REFERENCES}

Achmad, B., M.M. Mustafa and A. Hussain, 2011. Warped optical-flow inter-frame reconstruction for ultrasound image enhancement. J. Comput. Sci., 7: 1532-1540. DOI: 10.3844/jcssp.2011.1532.1540

Chan, R.H., C.W. Ho and M. Nikolova, 2005. Salt-andpepper noise removal by median-type noise detectors and detail-preserving regularization. IEEE Trans. Image Proc., 14: 1479-1485. DOI: 10.1109/TIP.2005.852196

Chen, H., A. Li., L. Kaufman and J. Hale, 1994. A fast filtering algorithm for image enhancement. IEEE Trans. Med. Image, 13: 557-564. DOI: $10.1109 / 42.310887$
Dawood, F.A.A., R.W. Rahmat, M.Z. Dimon, L. Nurliyana and S.B. Kadiman, 2011. Automatic boundary detection of wall motion in twodimensional echocardiography images. J. Comput. Sci., 7: 1261-1266. DOI: 10.3844/jcssp.2011.1261.1266

Frosio, I. and N.A. Borghese, 2006. Real time enhancement of cephalometric radiographies. Proceedings of the IEEE International Symposium on Biomedical Imaging: Nano to Macro, Apr. 6-9, IEEE Xplore press, Arlington, VA., pp: 972-975. DOI: $10.1109 /$ ISBI.2006.1625082

Gonzalez, R.C. and R.E. Woods, 2008. Digital Image Processing. 1st Edn., Prentice Hall, Upper Saddle River, NJ., ISBN: 013168728X, pp: 954.

Jagatheeswari1, P., S.S. Kumar and M. Rajaram, 2009. Contrast enhancement for medical images based on histogram equalization followed by median filter. Proceedings of the International Conference on Man-Machine Systems. Oct. 11-13, Penang, Malaysia, pp 2A41-2A4 4.

Jaya, J. and K. Thanushkodi, 2011. Implementation of computer aided diagnosis system based on parallel approach of ant based medical image segmentation. J. Comput. Sci., 7: 291-297. DOI: 10.3844/jcssp.2011.291.297

Parveen, N.R.S. and M.M. Sathik, 2009. Enhancement of bone fracture images by equalization methods. Proceesings of the International Conference on Computer Technology and Development, Nov. 1315, IEEE Xplore Press, Kota Kinabalu, pp: 391394. DOI: $10.1109 /$ ICCTD.2009.115

Ping, W., J. Li., L. Dongming and C. Gang, 2006. A multi-scale enhancement method to medical images based on fuzzy logic. Proceedings of the $10^{\text {th }}$ Region Conference TENCON., Nov. 14-17, IEEE Xplore Press, Hong Kong, China, pp: 1-4. DOI: 10.1109/TENCON.2006.343785

Pizer, S.M., R.E. Johnston, J.P. Ericksen, B.C. Yankaskas and K.E. Muller, 1990. Contrast-limited adaptive histogram equalization: Speed and effectiveness. Proceedings of the 1st Conferance On Visualization Biomedical Computing, May 2225, IEEE Xplore Press, Atlanta, GA, USA., pp: 337-345. DOI: 10.1109/VBC.1990.109340

Sebri, A., J. Malek and R. Tourki, 2007. Automated breast cancer diagnosis based on gvf-snake segmentation, wavelet features extraction and neural network classification. J. Comput. Sci., 3: 600-607. DOI: $10.3844 /$ jcssp.2007.600.607 
Sen, A., L. Lan and K.R. Hoffmann, 2002. Improved coronary vessel tracking using non-linear filter enhanced X-ray angiograms. Proceedings of the 19th Annual International Conference on Engineering Medicine Biology Society, Oct. 30Nov. 2, IEEE XPlore Press, Chicago, IL , USA., pp: 573-576. DOI: 10.1109/IEMBS.1997.757674

Ramos, M.C.G. and C.M.V. Tahan, 2009. An assessment of the electric power quality and electrical installation impacts on medical electrical equipment operations at health care facilities. Am. J. Applied Sci., 6: 638-645. DOI: 10.3844 /ajassp.2009.638.645.
Tsai, D.Y., Y. Lee, M. Sekiya, S. Sakaguchi and I. Yamada, 2002. A method of medical image enhancement using wavelet analysis. Proceedings of the 16th International Conference on Signal Processing, Aug. 26-30, IEEE Xplore Press, Japan, pp: 723-726. DOI: 10.1109/ICOSP.2002.1181158

$\mathrm{Xu}, \mathrm{Z}$., X. Liu and X. Chen, 2009. Fog removal from video sequences using contrast limited adaptive histogram equalization. Proceedings of the International Conference on Computaional Intellegence and Software Engineering, Dec. 11-13, IEEE Xplore Press, Wuhan, pp: 1-4. DOI: 10.1109/CISE.2009.5366207 